\title{
Schlanke Körper (Slim Fields)
}

\author{
Markus Junker \\ Mathematisches Institut \\ Eckerstrasse 1 \\ 79104 Freiburg, Germany \\ markus · junker@math . uni-freiburg. de
}

\author{
Jochen Koenigsmann \\ Mathematical Institute \\ 24-29 St Giles' \\ Oxford OX1 3LB, Great Britain \\ Jochen. Koenigsmann@unibas.ch
}

September 2007

\begin{abstract}
We examine fields in which model theoretic algebraic closure coincides with relative field theoretic algebraic closure. These are perfect fields with nice model theoretic behaviour. For example, they are exactly the fields in which algebraic independence is an abstract independence relation in the sense of Kim and Pillay. Classes of examples are perfect PAC fields, model complete large fields and henselian valued fields of characteristic 0 .
\end{abstract}

\section{Introduction and definition}

We consider fields, possibly with additional structure, that is we consider $\mathcal{L}$-structures satisfying the field axioms where $\mathcal{L}$ is a (possibly proper) extension of the ring language $\{0,1,+,-, \cdot\}$. Such a structure is called a pure field if every $\emptyset$-definable set is already $\emptyset$-definable in the ring language.

By $k^{\text {alg }}$ or $\widetilde{k}$, we denote the algebraic closure of $k$ in the field theoretic sense, and by $\operatorname{acl}(k)$ or $\operatorname{acl}_{K}(k)$ the model theoretic algebraic closure of $k$ in the structure $K$, that is the set of elements of $K$ which satisfy some formula with parameters from $k$ that is only satisfied by finitely many elements in $K$. The expressions "transcendental", "algebraically independent", and "relative algebraic closure" always refer to the field theoretic meaning. "Algebraic formula", "a formula algebraises an element" always refer to the model theoretic meaning.

Parameters, realisations of types, etc. are usually taken in a monster model (or in a sufficiently saturated elementary extension). In all cases, $\mathbb{F}_{0}$ stands for the prime field.

Definition 1.1 Let $K$ be a (not necessarily pure) field.

(a) The thickness of a subfield $k$ of $K$ is defined to be tr.d. $\left(\operatorname{acl}_{K}(k) / k\right)$.

(b) $K$ is called $\operatorname{slim}^{1}$ if the model theoretic algebraic closure in $K$ coincides with the relative field theoretic algebraic closure, i.e. if the thickness of all subfields of $K$ is zero.

(c) $K$ is called very slim if all fields that are elementarily equivalent to $K$ are slim.

The thickness of $k$ in $K$ and in $K^{*} \succcurlyeq K$ is the same, it only depends on $\operatorname{Th}(K)$ or, more precisely, on $\operatorname{tp}(k)$. Thus a slim field that realises all $n$-types over $\emptyset$ is very slim. In particular,

\footnotetext{
${ }^{1}$ in German: schlank
} 
to show that a field is very slim, it suffices to show that a sufficiently saturated model of its theory is slim.

In any case, $|\operatorname{acl}(k)| \leq|\mathcal{L}|+\aleph_{0}+|k|$. Therefore, the thickness of a countable subfield in a field with countable language (in particular in a pure field) is at most $\aleph_{0}$. For short, we speak of "thickness infinity".

First examples Well known examples of very slim fields are:

- Finite fields.

- Algebraically closed fields.

- Real closed fields.

- $p$-adically closed fields and their finite extensions.

- Perfect PAC fields.

Proof: This is obvious for finite fields. In the next three cases, the well known quantifier elimination results show that relatively algebraically closed subfields are elementary substructures. Finally, it is shown in [CMD] that the model theoretic algebraic closure in any PAC field equals the relative algebraic closure of the closure under $\lambda$-functions.

Contents Section 2 contains mainly abstract model theory. We show that the property of being very slim is equivalent to algebraic independence being an abstract independence relation in the sense of Kim and Pillay, and also that topologically defined independence (via the Zariski topology) coincides with algebraic independence. Moreover, we examine specific definability properties for varieties, providing another equivalent formulation of "very slimness". Section 3 introduces several classes of fields measuring their degree of "non-slimness". In Section 4, we examine the basic model theoretic and algebraic properties of slim fields: perfectness, behaviour of finite extensions, definable subfields, questions of axiomatisability. Section 5 contains our main algebraic results: Large model complete fields and henselian valued field of characteristic 0 are shown to be very slim. The paper finishes with some examples of fields that are not very slim.

\section{Independence relations}

An abstract independence relation in the sense of Kim and Pillay [KP], following Harnik and Harrington [HH], is a ternary relation $A \underset{C}{\downarrow} B$ (" $A$ is independent from $B$ over $C$ ") between parameter sets satisfying the properties: invariance under automorphisms, finite character, monotonicity and transitivity, symmetry, existence of free extensions ${ }^{2}$, and local character. Examples are "non-forking" in stable and more generally in simple theories (where the independence relation satisfies in addition the so-called independence theorem), independence in o-minimal theories, "equational independence" in almost equational theories [JuK], and "non-thorn-forking" in rosy theories [Ons].

Independence $\bar{a} \underset{C}{\downarrow} B$ in algebraically closed fields can be defined in two equivalent ways: as algebraic independence $\rfloor^{\text {lalg }}$, i.e. the transcendence degree of $\bar{a}$ over $C$ is the same as over $C \cup B$, or as topological independence $\rfloor^{\text {top }}$, i.e. the locus of $\bar{a}$ over $C \cup B$ contains an irreducible component

\footnotetext{
${ }^{2}$ The axiom is called "extension" in [KP], and "existence" in [Ad].
} 
of the locus of $\bar{a}$ over $C$. Non-forking in strongly minimal structures is a generalisation of algebraic independence, equational independence in almost equational theories a generalisation of topological independence. In arbitrary fields, topological independence satisfies all axioms of an abstract independence relation except possibly "symmetry", and algebraic independence all except possibly "existence" (this is of course only for the "real world", not for imaginaries).

Our first aim is to show the following result:

Theorem 2.1 Algebraic independence is an abstract independence relation in the above sense, if and only if the field is very slim, if and only if algebraic independence coincides with topological independence.

Proof: All axioms of an abstract independence relation except possibly "existence" always hold for algebraic independence. The existence axiom states that for given $A, B, C$ there is always an $A^{\prime} \equiv_{C} A$ with $A \underset{C}{\downarrow} B$. This immediately implies slimness: Suppose $a \in \operatorname{acl}(k)$ is transcendental over $k$. Let then $A$ consist of $a, B$ of all model theoretic conjugates of $a$ over $k$, and $C=k$. Then there is no realisation of $\operatorname{tp}(a / k)$ that is algebraically independent from $B$ over $k$, i.e. existence fails.

Conversely, we show that slimness implies the "existence axiom": Let $a, B, C$ be given. Ei-

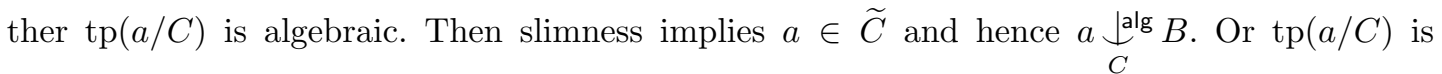
non-algebraic. Then among the infinitely many realisations, there must be an element that is transcendental over $B$. For finite tuples $\bar{a}$ the result follows by induction on the length $n$ of the tuple: First we find $\left(a_{1}^{\prime}, \ldots, a_{n}^{\prime}\right) \equiv_{C}\left(a_{1}, \ldots, a_{n}\right)$ with $a_{1}^{\prime} \underset{C}{\operatorname{lag}} B$ and then by induction $\left(a_{2}^{\prime \prime}, \ldots, a_{n}^{\prime \prime}\right) \equiv_{a_{1}^{\prime} C}\left(a_{2}^{\prime}, \ldots, a_{n}^{\prime}\right)$ with $a_{2}^{\prime \prime}, \ldots, a_{n}^{\prime \prime} \underset{C a_{1}^{\prime}}{\lfloor\text { alg }} B$. Together this yields $\left(a_{1}^{\prime}, a_{2}^{\prime \prime}, \ldots, a_{n}^{\prime \prime}\right) \equiv_{C}$ $\left(a_{1}, \ldots, a_{n}\right)$ and $a_{1}^{\prime}, a_{2}^{\prime \prime}, \ldots, a_{n}^{\prime \prime} \underset{C}{\lfloor\text { alg }} B$. For arbitrary $A$, the existence axiom follows by compactness.

For the second equivalence, if algebraic independence equals topological independence, then in particular algebraic independence satisfies the existence axiom, hence the field is very slim. Conversely, it is shown in [JuK], Example 3.8, that in very slim fields the two notions of independence coincide. This was done under the assumption of "almost strictness of the family of polynomial equations". We are defining this property in the next section and we prove an even stronger property for all fields in proposition 2.7.

Question 1 Find necessary and sufficient conditions for topological independence to satisfy "symmetry". Is this just "very slimness"?

The following is a test case for the general conjectures about stable and simple fields:

Question 2 If algebraic/topological independence is an independence relation with respect to which a field is stable (simple), is then the field (pseudo-)algebraically closed?

In particular, we may ask whether the following property of a field $K^{*}$ with enough automorphisms (e.g. saturated) implies PAC:

Whenever $K$ is a (small) elementary substructure of $K^{*}, L_{i}$ a field extension of $K$ in $K^{*}$ for $i=1,2, a_{i} \in K^{*}$ transcendental over $L_{i}$ such that $K\left(a_{1}\right)$ is isomorphic to 
$K\left(a_{2}\right)$ in $K^{*}$, then there exists an $a \in K^{*}$, transcendental over $L_{1} \cdot L_{2}$, such that $L_{1}(a)$ is isomorphic to $L_{2}(a)$ in $K^{*}$.

We know that a field satisfying this property can't be henselian valued w.r.t. a non-trivial valuation with residue characteristic 0 . If a conjecture of the second author is true, namely that any valued field with a non separably closed henselisation has a valuation that is definable in the ring language and induces the same topology, then all the henselisations of a field satisfying the above property are separably closed.

Hans Adler calls an abstract independence relation strict if the condition $a \underset{B}{\downarrow} a \Longrightarrow a \in \operatorname{acl}(B)$ is satisfied. (There is no connection with our notion of strictness to be defined in 2.2 below). Algebraic independence is always strict, as $a \underset{B}{\underset{\text { alg }}{a}} a$ implies $a \in \widetilde{B}$. Adler has shown in [Ad], Theorem 2.37, that if there is a strict independence relation at all on $T^{\mathrm{eq}}$, then the theory is rosy. Hence a theory of very slim fields is "rosy restricted to the home sort". Note that if a field is rosy with respect to algebraic independence, then it is automatically superrosy, as there are no infinite strictly descending forking chains then.

\section{Definability of varieties and strictness}

In this section we are going to prove the remaining ingredient for Theorem 2.1 and a new characterisation of very slim fields. Though in this article we are only interested in fields and polynomial equations, a more general approach helps to clarify the concepts. The terminology here follows mainly $[\mathrm{JuK}]$ where strictness in the sense below was introduced.

Let $\Gamma$ be a family of formulae $\varphi(\bar{x} ; \bar{y})$ in two sorts of variables: special variables $\bar{x}$ and parameter variables $\bar{y}$. A $\Gamma$-instance with parameters in $A$ is a formula $\varphi(\bar{x}, \bar{a})$ where $\varphi \in \Gamma, \bar{a}$ is from $A$, and the special variables remain free. A $\Gamma$-instance over $A$ is a formula $\varphi(\bar{x}, \bar{b})$ where $\varphi \in \Gamma, \bar{b}$ is arbitrary, and $\varphi(\bar{x}, \bar{b})$ is equivalent to a formula with parameters in $A$.

The closure of a family $\Gamma$ under positive boolean combinations and under renaming of variables, both respecting the sorts of the variables, will be denoted by $\langle\Gamma\rangle$. We use the notation $\doteq$ for the equality sign in formal languages. Note that if $x \doteq y$ is in $\Gamma$, then the $\Gamma$-instances are closed under adding mute parameters or variables. Otherwise this might not be the case. Usually, we are interested in familes closed under the two operations above and containing equality. We will call them normal. The following definition should be thought of being applied to normal families.

Definition 2.2 Let $\mathrm{cl}$ be an operator on parameter sets. A family of formulae $\Gamma$ is called clstrict if, for any parameter set $A$ (in the monster), any $\Gamma$-instance which is over $A$ is equivalent to a $\Gamma$-instance with parameters in $\operatorname{cl}(A)$.

"Strict" is short for "id-strict", and "almost strict" for "acl-strict".

If $\operatorname{cl}(A) \subseteq \operatorname{cl}^{\prime}(A)$ for all $A$, then cl-strictness implies cl'-strictness.

Example 2.3 (1) The family of all formulae is strict (this is by general model theoretic nonsense). The family of all Srour equations is strict ([Ju], Remark 2.2). The family of all stable formulae is strict (similar proof as for equations).

(2) The quantifier-free formulae are in general not strict: Consider for example formally real 
closed fields and the set defined by $x \doteq \sqrt{2}$ which is over $\emptyset$, but not quantifier-free definable without parameters.

(3) $\langle x \doteq y\rangle$ is cl-strict if and only if $\operatorname{acl}(A) \subseteq \operatorname{cl}(A)$ for all $A$.

Strictness of a family $\Gamma$ means that the two concepts "to be defined by a $\Gamma$-instance" and "to be over some parameter set" fit well together: Instead of working with $\Gamma$ one can as well work with the family of sets defined by $\Gamma$-instances.

The $\Gamma_{1}$-algebraic closure $\Gamma_{1}$-acl $(A)$ is the set of all elements algebraized by $\Gamma$-instances with parameters in $A$ (that is, the $\Gamma$-instance has only one free variable). In general it differs from the $\Gamma$-algebraic closure $\Gamma$-acl $(A)$ which is defined to be the set of all coordinates of tuples algebraized by $\Gamma$-instances with parameters in $A$.

Example 2.4 In a theory of an infinite field, let $\Pi$ be the family of all positive boolean combinations of polynomial equations with coefficients in the prime field (with all possible division of the variables into the two sorts). It is a normal family. Note that $\Pi_{1}-\operatorname{acl}(A)$ is just the relative algebraic closure of the subfield generated by $A$.

Lemma 2.5 If $\Gamma$ contains $x \doteq y$ and is cl-strict, then acl $\subseteq \Gamma_{1}$-acl $\circ$ cl.

Proof: Let $a \in \operatorname{acl}(B)$ have the conjugates $a_{1}, \ldots, a_{k}$. Then $x=a_{1} \vee \cdots \vee x=a_{k}$ is a $\Gamma$ instance over $B$, hence equivalent to some $\gamma(x, \bar{b})$ with $\gamma \in \Gamma$ and $\bar{b} \in \operatorname{cl}(B)$. This shows $a$ to be in $\Gamma_{1}-\operatorname{acl}(\operatorname{cl}(B))$.

An operator $\mathrm{cl}$ is called transitive if $\mathrm{clo} \mathrm{cl}=\mathrm{cl}$. The relative algebraic closure $\Pi_{1}$-acl is transitive.

Corollary 2.6 If $\Gamma$ contains $x \doteq y$ and is strict, then acl $=\Gamma_{1}$-acl.

If $\Gamma$ contains $x \doteq y$ and is $\Gamma_{1}$-acl-strict, and if $\Gamma_{1}$-acl is transitive, then acl $=\Gamma_{1}$-acl.

In general, an arbitrary normal family is not strict in any sense. However, polynomial equations in theories of fields are not far from being strict. For convenience, we will understand under variety in the rest of this section a subset of $K^{n}$ defined by a positive boolean combination of polynomial equations with coefficients in $K$, i.e. a relatively Zariski-closed set. No irreducibility is assumed.

The following is a variant of Weil's well known proof of the existence of a field of definition:

Proposition 2.7 In any theory of fields, $\Pi$ is dcl-strict.

Proof $^{3}$ : If $V \subseteq K^{n}$ is a variety that is $A$-invariant, let $M_{1}(\bar{X}), \ldots, M_{m}(\bar{X})$ be an enumeration of the monomials in $\bar{X}=\left(X_{1}, \ldots, X_{n}\right)$ that occur in the polynomials used to define $V$. To $V$, we associate the subspace

$$
U:=\left\{\bar{k} \in K^{m} \mid \sum_{i=1}^{m} k_{i} M_{i}(\bar{x})=0 \text { for all } \bar{x} \in K^{n}\right\} .
$$

Then $U$ is $A$-invariant. Let $\left\{e_{1}, \ldots, e_{m}\right\}$ be the canonical basis of $K^{m}$. Then we find in a canonical way a basis $\left\{f_{1}+U, \ldots, f_{l}+U\right\}$ of $K^{m} / U$ with $f_{i} \in\left\{e_{1}, \ldots, e_{m}\right\}$ : For example,

\footnotetext{
${ }^{3}$ We would like to thank Martin Ziegler for very helpful discussions.
} 
choose the smallest possible tuple of indices with respect to the lexicographical ordering. Thus we have unique expressions $e_{i}=\sum_{j} \alpha_{i, j} f_{j}+u_{i}$ with $u_{i} \in U$, and the $u_{i}$ are $A$-invariant, i.e. $u_{i} \in \operatorname{dcl}(A)$. Let $U^{\prime}=\left\langle u_{1}, \ldots, u_{l}\right\rangle$. Then all $e_{i}+U \in\left\langle f_{1}+U^{\prime}, \ldots, f_{l}+U^{\prime}\right\rangle$, hence the $f_{j}+U^{\prime}$ generate $K^{m} / U^{\prime}$, whence $U=U^{\prime}$. Now, because the $u_{i}$ generate $U$, the polynomials coded by the $u_{i}$ define $V$.

Proposition 2.8 In a theory of fields, $\Pi$ is strict iff the field is algebraically closed and has, up to interdefinability, no additional structure.

ProOF: " $\Leftarrow$ ": If a variety $V$ is defined over $A$, it can, by quantifier elimination, be written as a union of locally closed sets $V_{i} \backslash W_{i}$, where the $V_{i}, W_{i}$ are varieties defined by equations with coefficients from $A$. We may assume that the $V_{i}$ are " $A$-irreducible" and that $W_{i} \subset V_{i}$. But then $V_{i}$ equals the closure of $V_{i} \backslash W_{i}$, hence $V$ equals the union of the $V_{i}$.

" $\Rightarrow$ " Suppose $K$ is a sufficiently saturated field which is not algebraically closed or which has additional structure that is not definable in the field structure. Then there is an infinite, coinfinite $\bar{c}$-definable subset $T$ of $K$. Let $k=\mathbb{F}_{0}(\bar{c})$, choose elements $a \in T, b \notin T$ that are algebraically independent over $k$, and let $L=k(a+b, a b) . L$ is the fixed field of $L(a)=k(a, b)$ under the automorphism interchanging $a$ and $b$, therefore $a \notin L$ and $L(a) / L$ is a separable quadratic extension. Let $f$ be the minimal polynomial of $a$ and $b$ over $L$. Then the set $\{a\}$ is definable as a variety over $K$ by $x \doteq a$, it is definable over $L$ by $f(x) \doteq 0 \wedge x \in T$, but it is not definable as an $L$-variety, since $L$-polynomials can't distinguish between $a$ and $b$.

Proposition 2.9 A theory of fields is a theory of very slim fields if and only if $\Pi$ is $\Pi_{1}$-aclstrict, if and only if $\Pi$ is $\left(\Pi_{1}\right.$-acl $\left.\cap \mathrm{dcl}\right)$-strict.

PROOF: $\Pi$ is dcl-strict by proposition 2.7, hence also almost strict, and if model theoretic algebraic closure coincides with the relative algebraic closure, then $\operatorname{dcl}(A) \subseteq \operatorname{acl}(A)=\Pi_{1}-\operatorname{acl}(A)$, and dcl-strictness is the same as $\left(\Pi_{1}\right.$-acl $\left.\cap \mathrm{dcl}\right)$-strictness. The latter implies $\Pi_{1}$-acl-strictness by monotonicity. Finally, $\Pi_{1}$-acl is nothing else than relative algebraic closure and therefore transitive, hence the second part of corollary 2.6 shows that under $\Pi_{1}$-acl-strictness of $\Pi$, the two notions of algebraic closure coincide.

It might be interesting also to look at $\Pi$-acl. There are examples where it differs from the relative algebraic closure $\Pi_{1}$-acl. In the same way as above one sees that acl $=\Pi$-acl is a necessary condition for $\Pi$ to be $\Pi$-acl-strict in a theory of fields, and conversely that this implies acl $=\Pi$-acl $\circ \Pi$-acl. As there seems to be no reason for $\Pi$-acl to be transitive, a more natural class of fields to consider might be those where acl $=\Pi$-acl ${ }^{*}$, or equivalently where $\Pi$ is $\Pi$-acl*-strict for the transitive closure $\Pi$-acl ${ }^{*}$ of $\Pi$-acl. A priori, this is a larger class of fields than the very slim fields.

\section{Sizes of fields}

Let $K$ be a field. The body mass function $\mathrm{BM}_{K}: \mathbb{N} \rightarrow \mathbb{N} \cup\{\infty\}$ is defined to be

$$
\operatorname{BM}_{K}(n):=\sup \left\{\operatorname{tr} . \operatorname{d} .\left(\operatorname{acl}\left(a_{1}, \ldots, a_{n}\right) / a_{1}, \ldots, a_{n}\right) \mid a_{1}, \ldots, a_{n} \in K^{*} \succcurlyeq K\right\} .
$$

By definition, $\mathrm{BM}_{K}$ only depends on the elementary theory of $K$. We will often write BM for short. 
Thickness is in general not a monotonous function (all subfields of the form $\operatorname{acl}_{K}(k)$ have thickness zero in $K$ ), but the body mass function is: Choose an element $t$ that is transcendental over $\operatorname{acl}(k)$, then tr.d. $(\operatorname{acl}(k, t) / k(t)) \geqslant \operatorname{tr} . d .(\operatorname{acl}(k) / k)$. It follows that in the definition of the body mass function one could require $a_{1}, \ldots, a_{n}$ to be algebraically independent.

We use the body mass function to distinguish several types of theories of fields:

- Fields of size XS (or very slim fields): BM is the zero function.

- Fields of size S: BM is not the zero function, but bounded.

- Fields of size M: BM is unbounded, but linearly bounded (that is, BM is in $O(n)$ : eventually bounded by a linear function).

- Fields of size L: BM is not linearly bounded.

We consider the sizes as totally ordered in the usual sense: $\mathrm{XS}<\mathrm{S}<\mathrm{M}<\mathrm{L}$. A slim field might well have size $L$, but the size can be seen from the thicknesses of subfields of a sufficiently saturated model.

One is tempted to further divide the class $L$ and for example introduce $X L$ as those fields where $\mathrm{BM}$ is eventually infinite, and $X X L$ as those where the thickness of a subfield is actually infinite. Some of our proofs give additional results if one does so. But it not clear whether there are examples that would justify this distinction.

Remark 3.1 $\operatorname{acl}(A)=\widetilde{\operatorname{dcl}(A)} \cap K$ holds for any parameter set $A$ in any field $K$. In particular, $K$ is not slim if and only if there are definable transcendental elements over some finitely generated subfield. More precisely, whenever the thickness of $k \subseteq K$ is not zero, then there are definable elements over $k$ that are transcendental over $k$.

Proof: Let $t \in \operatorname{acl}_{K}(k) \backslash \tilde{k}$ for some finitely generated subfield $k \subseteq K$, and let $t_{1}, \ldots, t_{k}$ be the model theoretic conjugates of $t$ over $k$. Then $t$ is in the field theoretic sense algebraic over the elementary symmetric functions $s\left(t_{1}, \ldots, t_{k}\right)$ in the $t_{i}$. The $s\left(t_{1}, \ldots, t_{k}\right)$ are invariant over $k$, hence in $\operatorname{dcl}(k)$. The converse inclusion is trivial.

Thus the thickness of a subfield $k$ in $K$ equals tr.d. $(\operatorname{dcl}(k) / k)$.

Lemma 3.2 Let $K$ be a field. If in $K$ there is over $\bar{a}$ a definable transcendental which is algebraically independent from $\bar{a}$ over $\operatorname{dcl}(\emptyset)$, then $K$ has at least size $M$, that is, $K$ has subfields of arbitrarily big thickness.

Proof: Choose $(\bar{a}, t)$ of minimal length such that $t$ is definable over $\bar{a}$ and algebraically independent from $\bar{a}$ over $\operatorname{dcl}(\emptyset)$. By minimality, $\bar{a}=\left(a_{1}, \ldots, a_{k}\right)$ is algebraically independent over $\operatorname{dcl}(\emptyset)$, moreover $a_{i} \notin \operatorname{acl}(\emptyset)$ and $k \neq 0$. Let $p:=\operatorname{tp}(\bar{a}, t)$.

We are going to construct inductively a sequence $\left(\bar{a}_{n}, t_{n}\right)_{n \in \omega}$ of realisations of $p$ in such a way that $t_{n}$ is transcendental over $\bar{a}_{0}, \ldots, \bar{a}_{n}, t_{0}, \ldots, t_{n-1}$. Suppose the $(n+1)$ th step is not possible. This means that $p(\bar{x}, y) \vdash$ " $y \in \mathbb{F}_{0}\left(\bar{x}, \bar{a}_{0}, \ldots, \bar{a}_{n}, t_{0}, \ldots, t_{n}\right)^{\text {alg }}$ ", which implies by compactness that $p(\bar{x}, y) \vdash Q\left(y, \bar{x}, \bar{a}_{0}, \ldots, \bar{a}_{n}, t_{0}, \ldots, t_{n}\right) \doteq 0$ for a polynomial $Q$ that is not identical zero as polynomial in $y$ over $\mathbb{F}_{0}\left(\bar{x}, \bar{a}_{0}, \ldots, \bar{a}_{n}, t_{0}, \ldots, t_{n}\right)$. In particular, the zero set of $Q\left(y, \bar{x}, \bar{a}_{0}, \ldots, \bar{a}_{n}, t_{0}, \ldots, t_{n}\right)$ in $K^{k+1}$ has at most dimension $k$. It follows that, for the monster model $\mathbb{K}$,

$$
p(\bar{x}, y) \vdash \bigwedge_{\sigma \in \operatorname{Aut}(\mathbb{K})} Q\left(y, \bar{x}, \bar{a}_{0}^{\sigma}, \ldots, \bar{a}_{n}^{\sigma}, t_{0}^{\sigma}, \ldots, t_{n}^{\sigma}\right) \doteq 0 .
$$


By Proposition 2.7, the right part is equivalent to a positive boolean combination of polynomial equations with coefficients in $\operatorname{dcl}(\emptyset)$, and has (in the free variables $y, \bar{x}$ ) at most dimension $k$. Thus $p$ implies that $\bar{x}$ and $y$ are algebraically dependent over $\operatorname{dcl}(\emptyset)$ : contradiction.

By standard techniques we can make the sequence $\left(\bar{a}_{n}, t_{n}\right)_{n \in \omega}$ indiscernible in such a way that the property " $t_{n}$ is transcendental over $\bar{a}_{0}, \ldots, \bar{a}_{n}, t_{0}, \ldots, t_{n-1}$ " is preserved. As all fields are quantifier-free stable, any indiscernible sequence is an indiscernible set with respect to quantifier-free formulae. Hence $t_{i}$ is trancendental over $\bar{a}_{0}, \ldots, \bar{a}_{n}, t_{0}, \ldots, t_{i-1}, t_{i+1}, \ldots, t_{n}$ for every $i \in\{0, \ldots, n\}$. Thus the thickness of $\mathbb{F}_{0}\left(\bar{a}_{0}, \ldots, \bar{a}_{n}\right)$ is at least $n+1$.

Corollary 3.3 In fields of size $M$, the body mass function $\mathrm{BM}$ is in $\Theta(n)$, i.e. is eventually bounded from below and from above by non-constant linear functions.

ProOF: BM is at most growing linearly by definition, and at least like $n \mapsto\left\lceil\frac{n}{k}\right\rceil$ for some $k \geq 1$ according to the proof of Lemma 3.2.

Proposition 3.4 The body mass function is constant in fields of size at most S. Moreover, $\operatorname{acl}(\bar{a})=\mathbb{F}_{0}(\bar{a}, \operatorname{dcl}(\emptyset))^{\text {alg }}$ holds for all $\bar{a}$. In particular, in fields of size $S$ there are $\emptyset$-definable transcendentals.

Proof: Let $K$ be of size S. Thus there are $\bar{a}$ and $t \in \operatorname{acl}(\bar{a}) \backslash \mathbb{F}_{0}(\bar{a})^{\text {alg }}$. According to Lemma 3.2, $t$ is algebraically dependent from $\bar{a}$ over $\operatorname{dcl}(\emptyset)$. If there are no $\emptyset$-definable transcendentals, then $\operatorname{dcl}(\emptyset) \subseteq \mathbb{F}_{0}{ }^{\text {alg }}$, hence $t \in \mathbb{F}_{0}(\bar{a}, \operatorname{dcl}(\emptyset))^{\text {alg }} \subseteq \mathbb{F}_{0}(\bar{a})^{\text {alg }}$ : contradiction.

Now let $k$ be a finitely generated subfield of $K$. By Lemma 3.2, $\operatorname{dcl}(k)$ is an algebraic extension of $\mathbb{F}_{0}(k, \operatorname{dcl}(\emptyset))^{\text {alg }}$. Hence $\operatorname{acl}(k)=\operatorname{dcl}(k)^{\text {alg }} \cap K \subseteq \mathbb{F}_{0}(k, \operatorname{dcl}(\emptyset))^{\text {alg }}$. It follows that tr.d. $(\operatorname{acl}(k) / k) \leqslant$ tr.d. $(\operatorname{dcl}(\emptyset) / k) \leqslant \operatorname{tr} . d .(\operatorname{acl}(\emptyset))=\operatorname{BM}_{K}(0)$.

Proposition 3.4 implies that Theorem 2.1 can be modified for fields of size S: If we define algebraic independence over $\operatorname{dcl}(\emptyset)$ and topological independence over $\operatorname{dcl}(\emptyset)$ by " $\bar{a} \underset{C}{\downarrow} B$ in the new sense if and only if $\bar{a} \underset{C \cup}{\downarrow} B$ in the old sense", then algebraic independence over $\operatorname{dcl}(\emptyset)$ is an abstract independence relation if and only if it equals topological independence over $\operatorname{dcl}(\emptyset)$ if and only if the field has size S.

Example 3.5 (1) An algebraically closed field with constants for $n$ algebraically independent elements is an example of a (non pure) field of size S: BM is constant $n$.

(2) $(\mathbb{C}, x \mapsto \bar{x})$ is an example of a field of size $\mathrm{M}$ : Choose algebraically independent elements $r_{1}, \ldots, r_{2 n}$ in $\mathbb{R}$ and put $t_{j}:=r_{j}+\mathrm{i} r_{j+n}$. Then $r_{j}, r_{j+n} \in \operatorname{dcl}\left(t_{j}, \overline{t_{j}}\right)=\operatorname{dcl}\left(t_{j}\right)$. Thus the thickness of the subfield generated by $t_{1}, \ldots, t_{n}$ is at least $n$. It is not difficult to see on the other hand that $\operatorname{BM}(n) \leqslant n$. (Or use Lemma 4.5 to see that this field has at most size $\mathrm{M}$ ).

Question 3 Are there pure fields of size S? of size M?

\section{Properties of slim fields}

\section{Definable subfields and perfectness}

Proposition 4.1 Very slim fields are perfect and do not have proper infinite definable subfields. 
Finite fields are perfect, and as infinite fields have elementary extensions of arbitrarily high transcendence degree, the proposition follows from the following lemma:

Lemma 4.2 If $K$ has a proper $\bar{a}$-definable subfield $k$ for $\bar{a} \in K^{n}$, and if either $k$ has transcendence degree at least $n+2$ and $K$ is a non-algebraic extension of $k$, or $k$ has transcendence degree at least $n+3$, then $K$ is not slim.

Proof: Choose $t \in K \backslash k$ and $b_{0}, b_{1} \in k$ such that $t, b_{0}, b_{1}$ are algebraically independent over $\bar{a}$, and put $c:=b_{0}+b_{1} t$. Then $b_{0}, b_{1}$ are definable over $(\bar{a}, c, t)$ as the unique coefficients in $k$ of $c$ as a linear term in $t$, but the transcendence degree of $\left(b_{0}, b_{1}\right)$ over $(\bar{a}, c, t)$ is at least 1 , contradicting slimness.

Corollary 4.3 If $K$ is slim and has transcendence degree at least 3 , then $K$ is perfect.

This is best possible: Corollary 6.2 shows that $\mathbb{F}_{p}(X, Y)^{\mathrm{sep}}$ is slim.

The example of PAC fields shows that the adequate notion of slimness for non-perfect fields should be the following:

Definition 4.4 $K$ is $\lambda$-slim if the model theoretic algebraic closure in $K$ equals the relative algebraic closure of the closure under $\lambda$-functions.

Non-forking in separably closed fields can be described as algebraic independence of $\lambda$-closures. In analogy to Theorem 2.1 we get that algebraic independence of $\lambda$-closures is an abstract independence relation if and only if we are in a theory of $\lambda$-slim fields.

\section{Finite extensions}

Our list of examples of very slim pure fields is closed under finite extensions. This raises the question whether a finite extension of a very slim field is again very slim. On the other hand, the structure $(\mathbb{C}, x \mapsto \bar{x})$ of size $\mathrm{M}$ and with $\operatorname{BM}(x)=2 x$ is interpretable in the very slim field $\mathbb{R}$. We will show now that this is the worst case: No finite field extension of a very slim field can have size L. For this, we will understand the following under a finite field extension $L / K$ for fields with possibly additional structure: The extra structure on $L$ should be induced by the structure on $K$ via the standard interpretations of the pure field $L$ in the pure field $K$.

Lemma 4.5 Let $L / K$ be a finite field extension of degree $n$. Then

$$
\mathrm{BM}_{K}(n(m+1)) \geqslant \mathrm{BM}_{L}(m)+m-m n-n .
$$

Proof: Let $L=K(\alpha)$ and let $\bar{b}$ be the $n$ coefficients of the minimal polynomial of $\alpha$ over $K$. Then there is a $\bar{b}$-definable field structure isomorphic to $L$ on $K^{n}$, and by the assumption on the additional structure, we may assume that the whole structure on $L$ lives $\bar{b}$-definably on $K^{n}$. Let $\chi: L \rightarrow K^{n}$ be the corresponding isomorphism, and let $\bar{\chi}: \mathfrak{P}(L) \rightarrow \mathfrak{P}(K)$ be the map that sends $X \subseteq L$ to the set of all coordinates of $\chi(x)$ for all $x \in X$. Since the primitive element $\alpha$ is in $\mathbb{F}_{0}(\bar{b})^{\text {alg }}$, any subfield $l$ of $L$ is algebraic over $\bar{\chi}[l]$ and $\bar{b}$ in the field theoretic sense.

Consider now a subfield $l$ of $L$ generated by $m$ elements $g_{1}, \ldots, g_{m}$ and of thickness $\operatorname{BM}_{L}(m)$, or of arbitrarily high thickness if $\mathrm{BM}_{L}(m)=\infty$. We may assume that $l$ has transcendence degree $m$. We want to compute the thickness of $k:=\mathbb{F}_{0}(\bar{\chi}[l], \bar{b})$. 
On the one hand, one sees that $\bar{\chi}[l]$ lies in the field generated by $\bar{\chi}\left[\left\{g_{1}, \ldots, g_{m}\right\}\right]$ and $\bar{b}$, hence tr.d. $(k) \leq m n+n$. On the other hand, note that $\bar{\chi}\left[\operatorname{acl}_{L}(l)\right] \subseteq \operatorname{acl}_{K}(\bar{\chi}[l], \bar{b})=\operatorname{acl}_{K}(k)$ because $L$ is interpretable in $K$ over $\bar{b}$. Therefore

$$
\operatorname{tr.d.}\left(\operatorname{acl}_{K}(k)\right) \geq \operatorname{tr} . \text { d. }\left(\bar{\chi}\left[\operatorname{acl}_{L}(l)\right] \cup \bar{b}\right) \geq \operatorname{tr} . \text { d. }\left(\operatorname{acl}_{L}(l)\right)=\mathrm{BM}_{L}(m)+m .
$$

Put together, the thickness of $k$ is at least $\mathrm{BM}_{L}(m)+m-m n-n$. Because the body mass function is monotonous, the lemma follows.

Proposition 4.6 Let $L / K$ be a finite extensions of fields of degree $n$. If $K$ has size at most $M$, then also L. More precisely: If $\mathrm{BM}_{k}$ is eventually bounded by $m \mapsto \alpha m$, then $\mathrm{BM}_{L}$ is eventually bounded by $m \mapsto(n \alpha+n) m$.

Proof: Use Lemma 4.5 and a little computation for (b).

The example of $\mathbb{R}$ and $(\mathbb{C}, x \mapsto \bar{x})$ shows that this is at least not far from being best possible for fields with additional structure. We have no example of a pure finite field extension of a very slim field that is not very slim, but a priori there is no reason why a similar situation should not occur definably in some pure field. (But compare with Proposition 4.1.)

Question 4 Is a finite pure field extension of a very slim field again very slim? Is a field very slim if some finite extension of it is very slim?

$\mathrm{Al}$ least, we can show that a finite extension of a very slim field $K$ is " $K$-slim":

Proposition 4.7 Let $L / K$ be a finite extension, $K$ a very slim field. Then no formula in $L$ with parameters in $K$ can algebraize elements that are transcendental over these parameters.

Proof: Assume $c \in \operatorname{acl}_{L}(\bar{a})$ for $\bar{a}$ in $K$. If $K$ is finite, $c$ is in $\mathbb{F}_{0}(\bar{a})^{\mathrm{alg}}$. If $K$ is infinite, we may assume $K$ to be sufficiently saturated. We can find a primitive element $\alpha$ of $L / K$ such that the set $\alpha_{K}$ of non-leading coefficients of the minimal polynomial of $\alpha$ over $K$ is algebraically independent over $\mathbb{F}_{0}(c, \bar{a})$, see Lemma 4.8. Let $c_{\alpha}$ be the set of coefficients of $c$ w.r.t. the basis $\left\{1, \alpha, \alpha^{2}, \ldots\right\}$ of $L$ over $K$. Then $c_{\alpha} \subseteq \operatorname{acl}_{K}\left(\bar{a}, \alpha_{K}\right)$. Because $K$ is very slim, $c_{\alpha} \subseteq \mathbb{F}_{0}\left(\bar{a}, \alpha_{K}\right)^{\text {alg }}$,

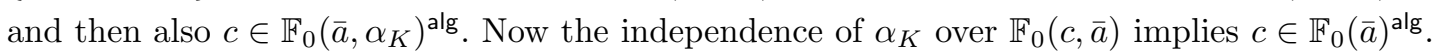

The lemma we need to complete the proof might be well-known to algebraists:

Lemma 4.8 Let $K$ be a field of infinite transcendence degree over its prime field $\mathbb{F}_{0}$, let $L / K$ be a finite separable extension and let $C$ be a finite subset of the algebraic closure $\widetilde{K}$ of $K$. Denote the set of coefficients of the minimal polynomials of the elements of $C$ over $K$ by $C_{K}$.

Then there is a primitive element $\alpha$ for $L / K$ such that the non-leading coefficients of the minimal polynomial of $\alpha$ over $K$ are algebraically independent over $\mathbb{F}_{0}\left(C_{K}\right)$, and hence also over $\mathbb{F}_{0}(C)$.

Proof: Pick some primitive element $\pi$ for $L / K$, let $n:=[L: K]$ and let $\pi_{K}$ be the set of coefficients of the minimal polynomial of $\pi$ over $K$. Choose $a_{0}, \ldots, a_{n-1} \in K$ algebraically independent over $\mathbb{F}_{0}\left(C_{K} \cup \pi_{K}\right)$, let $A:=\left\{a_{0}, \ldots, a_{n-1}\right\}$ and define $\alpha:=a_{0}+a_{1} \pi+\cdots+a_{n-1} \pi^{n-1}$. 
Let $\sigma_{1}, \ldots, \sigma_{n}$ be the distinct embeddings of $L$ into $\widetilde{K}$ over $K$, let

$$
f(X):=\prod_{i=1}^{n}\left(X-\sigma_{i}(\alpha)\right)=: X^{n}+b_{n-1} X^{n-1}+\cdots+b_{0} \in K[X]
$$

and let $B:=\left\{b_{0}, \ldots, b_{n-1}\right\}$. Then $f$ is irreducible over $K$, since $\sigma_{i}(\alpha) \neq \sigma_{j}(\alpha)$ for $i \neq j$ : otherwise

$$
a_{1}\left(\sigma_{j}(\pi)-\sigma_{i}(\pi)\right)+a_{2}\left(\sigma_{j}\left(\pi^{2}\right)-\sigma_{i}\left(\pi^{2}\right)\right)+\cdots+a_{n-1}\left(\sigma_{j}\left(\pi^{n-1}\right)-\sigma_{i}\left(\pi^{n-1}\right)\right)=0
$$

and hence $a_{1}, \ldots, a_{n-1}$ are linearly dependent over $\mathbb{F}_{0}\left(\sigma_{1}(\pi), \ldots \sigma_{n}(\pi)\right)$ and therefore algebraically dependent over $\mathbb{F}_{0}\left(\pi_{K}\right)$ - a contradiction.

The equation $f(\alpha)=0$ translates into $n$ polynomial equations over $\mathbb{F}_{0}\left(B, \pi_{K}\right)$ for the coefficients $a_{0}, \ldots, a_{n-1}$ of $\alpha$ w.r.t. the base $1, \pi, \ldots, \pi^{n-1}$ of $L$ over $K$. This way $A$ can be seen to be algebraic over $\mathbb{F}_{0}\left(B \cup \pi_{K}\right)$. By the choice of $A$,

$$
\text { tr.d. }\left(\mathbb{F}_{0}\left(A \cup C_{K} \cup \pi_{K}\right) / \mathbb{F}_{0}\left(C_{K} \cup \pi_{K}\right)\right)=n=|A|=|B| .
$$

Hence

$$
\text { tr.d. }\left(\mathbb{F}_{0}\left(B \cup C_{K} \cup \pi_{K}\right) / \mathbb{F}_{0}\left(C_{K} \cup \pi_{K}\right)\right)=n,
$$

i.e. $B$ is algebraically independent over $\mathbb{F}_{0}\left(C_{K} \cup \pi_{K}\right)$, and so, in particular, over $\mathbb{F}_{0}\left(C_{K}\right)$.

One can show for arbitrary fields that finite extensions do not add "new" $\emptyset$-definable transcendentals:

Lemma 4.9 Let $L / K$ be a finite extension of pure fields. Then $\operatorname{dcl}_{L}(\emptyset) \cdot \operatorname{dcl}_{K}(\emptyset)$ is an algebraic extension of $\mathrm{dcl}_{K}(\emptyset)$.

Proof: Let $c \in L$ be $\emptyset$-definable, and let $M$ be the normal hull of $L$ in a fixed algebraic closure $\widetilde{L}$. We may suppose $K$ to be a saturated model. Then $L$ and $M$ are saturated, too. As $L$ has only finitely many embeddings into $M$; the orbit of $c$ under $\operatorname{Aut}(M)$ is finite. As $M$ is saturated, $c$ is model theoretically algebraic in $M$. By remark 3.1, we may assume $c$ to be $\emptyset$-definable in $M$. Now every automorphism of $K$ lifts to an automorphism of $M$ and thus fixes $c$. It follows that every automorphism fixes the minimal polynomial of $c$ over $K$. Again by saturation, the coefficients are in $\operatorname{dcl}_{K}(\emptyset)$, that is, $c$ is in the relative algebraic closure of $\operatorname{dcl}_{K}(\emptyset)$ in $L$.

Corollary 4.10 If a pure finite field extension of a very slim field is not very slim, then it has size $M$.

The finite extension property is false for slim fields: $\mathbb{Q}(X)$ is slim (Example 6.3), but functions fields over $\mathbb{Q}$ of curves of genus higher than 1 are not slim (Example 6.6).

\section{Interpretations}

Reducts of slim fields (with extra structure) are slim. More generally, the size of a reduct is at most the size of the original structure. Naming constants can increase the size of very slim fields to size $\mathrm{S}$ if the constants are transcendental. In all other cases, the size remains unchanged.

Lemma 4.11 Elementary substructures of slim fields are slim. 
Proof: Assume $K$ is not slim, say $t \in \operatorname{acl}_{K}(k) \backslash \widetilde{k}$ for some subfield $k$ of $K$. Then for $K^{*} \succcurlyeq K$ we get on the one hand $t \in \operatorname{acl}_{K}(k)=\operatorname{acl}_{K^{*}}(k)$, on the other hand $t \notin \widetilde{k} \cap K^{*}=\widetilde{k} \cap K$ as $K$ is relatively algebraically closed in $K^{*}$. Thus $K^{*}$ is not slim.

Lemma 4.12 Let $K$ be a field with a proper infinite $\bar{c}$-definable subfield L. (Thus $K$ is not very slim by Proposition 4.1.)

(a) $\mathrm{BM}_{L}(x) \leqslant \mathrm{BM}_{K}(x+\lg (\bar{c}))+\operatorname{tr} . \mathrm{d} .(\bar{c})$. Hence $L$ has at most the size of $K$.

(b) If $K$ is a non-algebraic extension of $L$, then $K$ has size $L$.

(c) If $K$ is an algebraic extension of $L$, then $K$ has at least size $M$.

A stupid counterexample to a stronger version: Let $K$ be very slim and $L$ the expansion of $K$ by constants for algebraically independent elements $\bar{c}$.

ProOF: Going up to elementary extensiosn, we may suppose $L$ and $K$ to be sufficiently saturated, in particular to have infinite transcendence degree.

(a) Let $L$ be definable in $K$. If there is a formula that in $L$ algebraises a transcendental element $t$ over $l \subseteq L$, then in $K$ the same formula with quantifiers relativised to $L$ algebraises $t$ over $l$ and $\bar{c}$. That is, $\operatorname{acl}_{L}(l) \subseteq \operatorname{acl}_{K}(l, \bar{c})$. It follows that

$$
\operatorname{tr.d.}\left(\operatorname{acl}_{K}(l, \bar{c}) / l, \bar{c}\right) \geqslant \operatorname{tr} . d .\left(\operatorname{acl}_{K}(l, \bar{c}) / l\right)-\operatorname{tr} . d .(\bar{c}) \geqslant \operatorname{tr.d.}\left(\operatorname{acl}_{L}(l) / l\right)-\operatorname{tr} . d .(\bar{c}) .
$$

We can then choose for any $m$ an $l$ such that the thickness of $l$ equals $\operatorname{BM}_{L}(m)$.

(b) Choose elements $b_{i} \in L$ and $t \in K \backslash L$ that are algebraically independent over $\bar{c}$, and put $c_{m}:=b_{0}+b_{1} t+\cdots+b_{m} t^{m}$. Then the thickness of $\mathbb{F}_{0}\left(\bar{a}, c_{m}, t\right)$ is at least $m+1$ as $b_{0}, \ldots, b_{m}$ are definable over $\bar{a}, c_{m}, t$.

(c) Choose elements $a_{i}, b_{i}$ in $L$, algebraically independent over $\bar{c}$, and $t \in K \backslash L$. Then the thickness of $\mathbb{F}_{0}\left(\bar{c}, a_{0} t+b_{0}, \ldots, a_{n} t+b_{n}\right)$ is at least $n+1$.

Essentially the same proof shows that a field is not slim if a non-slim field is $\emptyset$-definable in it.

Corollary 4.13 If $K$ is a field of characteristic 0 in which $\mathbb{Q}$ is definable, then $K$ has size $L$.

This comes from the fact that $\mathbb{Q}$ has size $\mathrm{L}$, see Example 6.4. Therefore number fields, $\mathbb{Q}(X)$ and $\mathbb{R}(X)$ have size $\mathrm{L}$ by well-known results of Julia Robinson, see [Rob1] and [Rob2].

In general, nothing can be said about the size of a field $K$ compared to the size of a field interpretable in $K$ because the interpretation does not need to be compatible with the field structure.

\section{Axiomatisability}

Lemma 4.14 "Very slim" is a $\forall \exists$-property. That means, if $K$ is very slim, and $L \equiv \forall \exists K$, then $L$ is very slim.

Proof: Let $K$ be slim. Then for each formula $\varphi$, all parameters $\bar{c}$ and each $n$, there is an $m$ such that

$$
K \vDash \forall x\left(\left(\exists^{=n} z \varphi(z, \bar{c}) \wedge \varphi(x, \bar{c})\right) \rightarrow \exists z_{0} \ldots z_{m}\left(\bigwedge_{i=0}^{m} z_{i} \in \mathbb{F}_{0}(\bar{c}) \wedge z_{m} \neq 0 \wedge \sum_{i=0}^{m} z_{i} x^{i}=0\right)\right) .
$$


If $K$ realises all $k$-types, then for fixed $\varphi$ and $n$, the number $m$ as well as the complexity of the $z_{i}$ as terms in $\bar{c}$ is bounded, because otherwise one could realise the type of $(a, \bar{c})$ with $a$ transcendental over $\mathbb{F}_{0}(\bar{c})$, but $\varphi(a, \bar{c})$ and $|\varphi(x, \bar{c})|=n$ hold. Hence there is an $m$ and finite sets $\mathcal{T}_{0}^{i}$ of termes such that

$$
K \vDash \forall \bar{y} \forall x\left(\left(\exists^{=n} z \varphi(z, \bar{y}) \wedge \varphi(x, \bar{y})\right) \rightarrow \exists z_{0} \ldots z_{m}\left(\bigwedge_{i=0}^{m} \bigvee_{\tau \in \mathcal{T}_{0}^{i}} z_{i}=\tau(\bar{y}) \wedge \sum_{i=0}^{m} z_{i} x^{i}=0\right)\right)
$$

This is a $\forall \exists$-formula since $\exists^{=n} z$ is a $\Delta_{2}$-quantifier.

Remark 4.15 (1) The lemma does not imply that "very slim" is an elementary property, because the bounds for the complexity could be arbitrarily big for different very slim fields. And for the same reason, the lemma doesn't show that unions of increasing chains of very slim fields are very slim. This holds however if the fields in the chain are elementarily equivalent to each other.

(2) "Very slim" is not $\Delta_{2}$, because a non-perfect separably closed field $K$ is not very slim, but its algebraic closure, which is very slim, can be written as a union of an increasing chain of isomorphic copies of $K$ and thus has the same $\Delta_{2}$-theory as $K$.

It is even so that for every field $K$ there is a function field in one variable $F$ over $K$ such that $K$ is not algebraically closed in $F$ in the model theoretic sense, see [Koe].

Question 5 Is "very slim" an elementary property?

Clearly, it is not finitely axiomatisable, because "not very slim" is not an elementary property: An ultraproduct of non-perfect separably closed fields of varying characteristic is algebraically closed, hence very slim.

\section{Classes of very slim fields}

\section{Quantifier elimination and model completeness}

For a field in the ring language, to be very slim is a kind of quantifier elimination condition for algebraic formulae: The field is very slim if and only if each algebraic formula implies a quantifier-free algebraic formula with same parameters. (Look at $x^{2} \doteq 2 \wedge \exists y y^{2} \doteq x$ in $\mathbb{R}$ to see that "implies" can't be replaced by "equals" and that it is necessary to stick to the pure ring language here).

Remark 5.1 Every field is quantifier-free slim, i.e. it is not possible that transcendental elements are algebraised by quantifier-free formulae.

This is clear for finite fields as they are very slim. For infinite fields, a slightly generalised proof shows:

Proposition 5.2 Assume $K$ elimininates quantifiers in an extension $\mathcal{L}$ of the ring language by relation symbols, and that for all relation symbols $R_{i}$ in $\mathcal{L}$ and all polynomials $P_{i} \in K[X]$, each consistent expression ${ }^{4}$

$$
(\neg) R_{i_{1}}\left(P_{1}(x), \bar{a}_{1}\right) \wedge \cdots \wedge(\neg) R_{i_{n}}\left(P_{n}(x), \bar{a}_{n}\right)
$$

\footnotetext{
${ }^{4}$ The negation symbols in parentheses stands for two possibilities: either a negation symbol or nothing.
} 
defines an infinite set in $K$. Then $K$ is slim.

ProOF: Let $\varphi(x, \bar{a})$ be a quantifier-free algebraic formula. We may assume that it is a conjunction of atomic and negated atomic formulae. If among the atomic ones there is a polynomial equation, then this equation shows every realisation of $\varphi(x, \bar{a})$ to be field theoretically algebraic over $\bar{a}$. Otherwise the formula has the form

$$
\bigwedge_{j} Q_{j}(x, \bar{a}) \neq 0 \wedge(\neg) R_{i_{1}}\left(P_{1}(x), \bar{a}_{1}\right) \wedge \cdots \wedge(\neg) R_{i_{n}}\left(P_{n}(x), \bar{a}_{n}\right)
$$

for polynomials $Q_{j} \in \mathbb{F}_{0}[X]$. Now the first conjunct defines a co-finite set, therefore the whole formula defines an infinite set by our hypothesis.

Example 5.3 Proposition 5.2 applies immediately to algebaically closed fields and to real closed fields. By results of Kuhlmann, it will apply to $K((X))$ for an algebraically closed field $K$ of characteristic 0 , but these fields are shown to be very slim in Theorem 5.5 .

\section{Large fields}

A field $K$ is large if $K$ is existentially closed in the formal power series field $K((X))$, or equivalently, if every smooth curve with a $K$-rational point has infinitely many $K$-rational points, cf. [Pop]. Slimness is a similarly defined condition: A consistent formula in one variable which does not imply a non-trivial polynomial equation has infinitely many solutions.

Theorem 5.4 Pure fields that are large and model complete are very slim.

Proof: Let $K$ be model complete and large.

1. By model completeness, $K$ is perfect: $K \cong K^{p} \subseteq K$, hence $K^{p} \preccurlyeq K$, hence $K^{p}=K$.

2. The setting: Let $k \subseteq K$ be a subfield and let $\varphi(x)$ be a formula with parameters from $k$ and with one free variable $x$ such that $\{c \in K \mid K \vDash \varphi(c)\}=\left\{c_{1}, \ldots, c_{n}\right\}$ is non-empty and finite. We have to show that $c_{1}, \ldots, c_{n} \in \tilde{k}$. Assume the contrary, say, that $c:=c_{1}$ is transcendental over $k$. This assumption will lead to a contradiction.

3. Standard reductions: Since $K$ is model complete, $\varphi(x)$ is equivalent to an existential formula $\exists y_{1} \cdots \exists y_{r} \psi\left(x, y_{1}, \ldots, y_{r}\right)$ in $K$, where $\psi$ is quantifier free with parameters from $k$, i.e. of the shape

$$
\psi\left(x, y_{1}, \ldots, y_{r}\right)=\bigvee_{i} \bigwedge_{j_{i}}(\neg) f_{i j_{i}}\left(x, y_{1}, \ldots, y_{r}\right) \doteq 0
$$

for some polynomials $f_{i j_{i}} \in k\left[X, Y_{1}, \ldots, Y_{r}\right]$.

By assumption, $\varphi(c)$ holds in $K$, so we can choose $d_{1}, \ldots, d_{r} \in K$ such that $\psi\left(c, d_{1}, \ldots, d_{r}\right)$ holds and we can choose an index $i$ for which $\bigwedge_{j}(\neg) f_{i j_{i}}\left(c, d_{1}, \ldots, d_{r}\right)=0$, thus assuming w.l.o.g. that $\psi$ is of the shape $\bigwedge_{j}(\neg) f_{j}\left(x, y_{1}, \ldots, y_{r}\right) \doteq 0$.

At the cost of extra existential quantifiers, we can also get rid of inequalities: For any polynomial $f(\bar{x})$ over any field $F$ one has

$$
f(\bar{x}) \neq 0 \Longleftrightarrow \exists z(z \cdot f(\bar{x})=1)
$$

Finally, we can reduce $\psi$ to just one polynomial equation: By our assumption, $K$ is not very slim and hence not algebraically closed, so there is some irreducible monic polynomial $g=$ 
$T^{d}+a_{d-1} T^{d-1}+\ldots+a_{0} \in K[T]$ of degree $d>1$. Passing (if necessary) to some saturated extension of $K$ we may choose $g$ such that $c$ remains transcendental over the subfield of $K$ generated over $k$ by the coefficients of $g$. In other words, we may assume $g \in k[T]$. Note that $a_{0} \neq 0$, since $g$ has no zero in $K$, and that the polynomial

$$
Z^{d} \cdot g(1 / Z)=a_{0} Z^{d}+a_{1} Z^{d-1}+\ldots+a_{d-1} Z+1
$$

also has no zero in $K$. Now let $\hat{g}(T, Z):=T^{d}+a_{d-1} T^{d-1} Z+\ldots+a_{0} Z^{d}$ be the homogenization of $g$. Then for any $t, z \in K$ one has

$$
\hat{g}(t, z)=0 \Longleftrightarrow t=z=0,
$$

thus encoding two equations ( $t=0$ and $z=0$ ) in one. This allows to inductively reduce $\varphi(x)$ to a formula of the shape

$$
\exists y_{1} \cdots \exists y_{r} f\left(x, y_{1}, \ldots, y_{r}\right) \doteq 0
$$

for some $f \in k\left[x, y_{1}, \ldots, y_{r}\right]$ for which $f\left(c, d_{1}, \ldots, d_{r}\right)=0$. (This last reduction to one polynomial is not really needed for the proof, it's just for beauty and for the fun of it.)

4. Basic algebra: Since $\varphi(x)$ holds only for finitely many elements in $K$, the variable $x$ occurs in $f\left(x, y_{1}, \ldots, y_{r}\right)$ and $c$ is in $k\left(d_{1}, \ldots, d_{r}\right)^{\text {alg }}$. Since $c$ is transcendental over $k$ some of the $d_{i}$ are also transcendental over $k$. After rearranging the $d$ 's we may assume that, for some $s \leqslant r$, $d_{s}, d_{s+1}, \ldots, d_{r}$ is a transcendence base of $F:=k\left(c, d_{1}, \ldots, d_{r}\right)$ over $k$.

Now let $E:=k\left(d_{s}, \ldots, d_{r}\right)-$ so $F / E$ is finite and $E / k$ purely transcendental -, and let $\alpha \in F$ be a primitive element for the maximal separable subextension of $F / E-$ so $E(\alpha) / E$ is separable and $F / E(\alpha)$ is purely inseparable. Let $h \in E[Z]$ be the irreducible polynomial of $\alpha$ over $E$, and let $l \geqslant 0$ be a sufficiently large integer such that $c^{p^{l}}, d_{1}^{p^{l}}, \ldots, d_{s-1}^{p^{l}} \in E(\alpha)$, where $p:=\max \{1, \operatorname{char} K\}$.

5. The trick: Now let $K\left(\left(t^{p^{l}}\right)\right)$ be the field of formal Laurent series in $t^{p^{l}}$ and choose elements $t_{s}, t_{s+1}, \ldots, t_{r} \in t^{p^{l}} K\left[\left[t^{p^{l}}\right]\right]$ which are algebraically independent over $K$, and let $\tilde{d}_{i}:=d_{i}+t_{i}$ for $s \leqslant i \leqslant r$. Then $\tilde{d}_{s}, \ldots, \tilde{d}_{r}$ are algebraically independent over $K$, and hence also over $k\left(d_{1}, \ldots, d_{r}\right)$ and over $k$. Let

$$
\chi_{0}: E=k\left(d_{s}, \ldots, d_{r}\right) \longrightarrow \widetilde{E}:=k\left(\tilde{d}_{s}, \ldots, \tilde{d}_{r}\right)
$$

be the isomorphism with $\left.\chi_{0}\right|_{k}=\operatorname{id}_{k}$ and $\chi_{0}\left(d_{i}\right)=\tilde{d}_{i}$ for $s \leqslant i \leqslant r$. Then $\chi_{0}^{-1}$ is the restriction of the $t^{p^{l}}$-adic place $\varrho$ on $K\left(\left(t^{p^{l}}\right)\right)$ to $\widetilde{E}$. Let $\tilde{h}$ be the image of $h$ under the canonical prolongation of $\chi_{0}$ to an isomorphism $E[Z] \rightarrow \widetilde{E}[Z]$. Then $\tilde{h} \in\left(K\left[\left[t^{p^{l}}\right]\right]\right)[Z]$ and $\varrho(\tilde{h})=h$.

Since $\alpha$ is a simple zero of $h$ in $K$, there is, by Hensel's Lemma, a unique zero $\tilde{\alpha} \in K\left[\left[t^{p^{l}}\right]\right]$ of $\tilde{h}$ above $\alpha$. By construction, the isomorphism $\chi_{0}$ extends to an isomorphism $\chi_{1}: E(\alpha) \rightarrow \widetilde{E}(\tilde{\alpha})$ with $\chi_{1}(\alpha)=\tilde{\alpha}$, and further to an isomorphism

$$
\chi: F \rightarrow \widetilde{F}:=\widetilde{E}\left(\tilde{\alpha}, \tilde{c}, \tilde{d}_{1}, \ldots, \tilde{d}_{s-1}\right)
$$

with $\chi(c)=\tilde{c}$ and $\chi\left(d_{i}\right)=\tilde{d}_{i}$, where $\tilde{c}:=\sqrt[p^{l}]{\chi_{1}\left(c^{p^{l}}\right)}$ and $\tilde{d}_{i}:=\sqrt[p^{l}]{\chi_{1}\left(d_{i}^{p^{l}}\right)}$ for $i=1, \ldots, s-1$. Note that $\widetilde{E}(\tilde{\alpha}) \subseteq K\left(\left(t^{p^{l}}\right)\right)$ and that, by $K$ being perfect, $K\left(\left(t^{p^{l}}\right)\right)=(K((t)))^{p^{l}}$. Hence $\widetilde{F} \subseteq$ $K((t))$.

6. The harvest: Since $\chi$ is the identity on $k$ and since the polynomial $f$ constructed at the end of step 4 is defined over $k$,

$$
f\left(\tilde{c}, \tilde{d}_{1}, \ldots, \tilde{d}_{r}\right)=f\left(\chi(c), \chi\left(d_{1}\right), \ldots, \chi\left(d_{r}\right)\right)=\chi\left(f\left(c, d_{1}, \ldots, d_{r}\right)\right)=\chi(0)=0,
$$


so $\varphi(\tilde{c})$ holds in $K((t))$. On the other hand, by the choice of $\tilde{d}_{s}, \ldots, \tilde{d}_{r}$,

$$
\text { tr.d. } \widetilde{F} K / K=\text { tr.d. } \widetilde{F} / k=r-s+1
$$

and $\tilde{c}=\chi(c) \in \widetilde{F}$ is transcendental over $k$, so $\tilde{c}$ is also transcendental over $K$. In particular, $\tilde{c} \neq c_{1}, \ldots, \tilde{c} \neq c_{n}$ (cf. 2.). Hence

$$
K((t)) \vDash \exists x\left(\varphi(x) \wedge \bigwedge_{j=1}^{n} x \neq c_{j}\right) .
$$

Since $K$ is large, it is existentially closed in $K((t))$, and the same formula holds in $K$. But this contradicts the original setting where $\{x \in K \mid K \vDash \varphi(x)\}=\left\{c_{1}, \ldots, c_{n}\right\}$.

There are large and very slim fields which are not model complete in the ring language, for example $\mathbb{C}((X))$. Other examples are non model-complete perfect PAC fields, see [Ax], [JWh], [Wh].

Question 6 Are there fields that are / Find fields that are:

(1) perfect and large, but not very slim;

(2) model complete (in the ring language), but not very slim;

(3) very slim, but neither large nor model complete;

(4) very slim and model complete (in the ring language), but not large.

A good candidate for a perfect large field that might not be very slim is the fixed field of a $p$-Sylow-subgroup of the absolute Galois group of $\mathbb{Q}$.

In this context, one should note a question by Macintyre: If $K$ is a model complete field, is then the absolute Galois group of $K$ small and does $K^{\text {alg }}=\mathbb{F}_{0}^{\text {alg }} \cdot K$ hold? Moreover, the second author thinks that a field with small absolute Galois group should be large. Together this would imply a model complete field to be large and would simplify Theorem 5.4 to: A model complete pure field is very slim. Also, this would provide a negative answer to (2) and (4) of the question above.

\section{Henselian fields}

Theorem 5.5 Any pure field of characteristic 0 with a non-trivial henselian valuation is very slim.

Recall that henselian valued fields are large!

Proof $^{5}$ : Let $K$ be a field of characteristic 0 admitting a non-trivial henselian valuation. We have to show that $K$ is very slim (in the pure ring language). So we take an arbitrary field $K^{\prime}$ which is elementarily equivalent to $K$ and we show that $K^{\prime}$ is slim.

1. We first observe that $K^{\prime}$ has an $\aleph_{1}$-saturated elementary extension $K^{\star}$ with a non-trivial henselian valuation $v$ of residue characteristic 0 :

Either $K$ is algebraically closed - then so is $K^{\prime}$ and any $\aleph_{1}$-saturated elementary extension $K^{\star}$ of $K^{\prime}$ has non-trivial valuations with residue characteristic 0 (since $K^{\star}$ is not algebraic over $\mathbb{Q})$ and all of them are henselian because $K^{\star}$ is algebraically closed.

\footnotetext{
${ }^{5}$ We would like to thank Angus Macintyre for very helpful discussions from which the main argument in the proof emerged.
} 
Or $K$ is not algebraically closed - then $K$ is not separably closed and hence allows a field theoretically definable henselian topology (by [PZ], section 7), a property which carries over to $K^{\prime}$ : The henselian topology on $K$ is uniformly definable on $K$ using as parameters from $K$ the coefficients of a separable irreducible polynomial over $K$. However, any other separable irreducible polynomial over $K$ induces the same topology. Hence the topology is $\emptyset$-definable in the language of rings and so $K^{\prime}$ is elementarily equivalent to $K$ even as topological field. So $K^{\prime}$ also carries a henselian topology. Then, again by [PZ] (Theorem 7.2), any $\aleph_{1}$-saturated elementary extension $K^{\star}$ of $K^{\prime}$ admits a non-trivial henselian valuation $v$. Passing, if necessary, to an $\aleph_{1}$-saturated elementary extension of $\left(K^{\star}, v\right)$ in the language of valued fields and replacing the valuation there by the finest (by saturation non-trivial) coarsening with residue characteristic 0 , we may assume that $\left(K^{\star}, v\right)$ has residue characteristic 0 . (Cf. for example [EP] for the notions of coarsenings and refinements of valuations).

By Lemma 4.11 it suffices to show that $K^{\star}$ is slim.

So replacing $K$ by $K^{\star}$, we may assume that $K$ is $\aleph_{1}$-saturated and that $K$ has a non-trivial henselian valuation $v$ with residue characteristic 0 . We have to show that, under these hypotheses, $K$ is slim.

2. Following $[\mathrm{Pas}]$, these hypotheses make sure that $(K, v)$ admits an angular component, i.e. a group homomorphism

$$
\text { ac : } K^{\times} \rightarrow k^{\times}
$$

coinciding on $\mathcal{O}_{v}^{\times}$with the residue map, where $k$ is the residue field and $\mathcal{O}_{v}$ the valuation ring of $(K, v)$. We consider $(K, v)$ as 3 -sorted structure $\mathcal{K}=(K, k, \Gamma)$ in the language $\mathcal{L}_{v f c}$ of valued fields with angular components which contains the associated maps $v: K^{\times} \rightarrow \Gamma(\Gamma$ is the value group of $v$ ) and ac : $K^{\times} \rightarrow k^{\times}$. Then, by [Pas], Theorem 4.1, $\mathcal{K}$ admits $K$-quantifier elimination, i.e. every $\mathcal{L}_{v f c}$-formula is equivalent to a formula where quantification occurs only w.r.t. the $k$ - and $\Gamma$-sort.

3. Now assume that $K$ is not slim. Then there is a $\mathcal{L}_{\text {ring }}$-formula $\varphi(\bar{t}, x)$ and parameters $\bar{a}$ from $K$ such that $\varphi(\bar{a}, x)$ defines a finite set in $K$ which is not in the algebraic closure of $\mathbb{F}_{0}(\bar{a})$. By $K$-quantifier elimination we may take $\varphi(\bar{t}, x)$ to be of the form

$$
\mathrm{Q}(\bar{y}, \bar{\gamma}) \bigvee_{k} \bigwedge_{l} \psi_{k l}(\bar{y}, \bar{\gamma}, \bar{t}, x)
$$

where $\mathrm{Q}(\bar{y}, \bar{\gamma})$ is a block of quantifiers over the tupel $\bar{y}$ of free variables in the $k$-sort and the tupel $\bar{\gamma}=\left(\gamma_{1}, \ldots, \gamma_{n}\right)$ of free variables in the $\Gamma$-sort, and where the formula $\psi_{k l}(\bar{y}, \bar{\gamma}, \bar{t}, x)$ is

$$
\begin{aligned}
& P_{k l}(\bar{t}, x)=0 \wedge R_{k l}(\bar{t}, x) \neq 0 \\
\wedge & f_{k l}\left(\operatorname{ac}\left(F_{k l}^{1}(\bar{t}, x)\right), \ldots, \operatorname{ac}\left(F_{k l}^{r_{k l}}(\bar{t}, x)\right), \bar{y}\right)=0 \\
\wedge & g_{k l}\left(\operatorname{ac}\left(G_{k l}^{1}(\bar{t}, x)\right), \ldots, \operatorname{ac}\left(G_{k l}^{s k l}(\bar{t}, x)\right), \bar{y}\right) \neq 0 \\
\wedge & \sum_{i_{k l}} \alpha_{i_{k l}} v\left(A_{i_{k l}}(\bar{t}, x)\right)+\sum_{j=1}^{n} \alpha_{j k l}^{\prime} \gamma_{j}=0 \\
\wedge & \sum_{i_{k l}} \beta_{i_{k l}} v\left(B_{i_{k l}}(\bar{t}, x)\right)+\sum_{j=1}^{n} \beta_{j k l}^{\prime} \gamma_{j}>0 .
\end{aligned}
$$

Here the various $P, R, F, G, A$ and $B$ are polynomials in $\mathbb{Z}[\bar{t}, x]$ (evaluated in $K$ ), the $f$ 's and $g$ 's are polynomials over $\mathbb{Z}$ (evaluated in $k$ ) and the $\alpha, \alpha^{\prime}, \beta, \beta^{\prime}$ are integers (occuring as scalars of the $\mathbb{Z}$-module $\Gamma$ ).

4. By assumption, we may pick some $x \in K$ satisfying $\varphi(\bar{a}, x)$, where $x$ is transcendental over $\mathbb{F}_{0}(\bar{a})$. Then none of the polynomials $P, R, F, G, A$ or $B$ becomes zero at $(\bar{a}, x)$, unless it is the 
zero-polynomial (in $x$ ). Hence we can choose a neighbourhood $U$ of $x$ such that replacing $x$ by any $x^{\prime} \in U$ changes the values of these polynomials by 1-units, i.e. by factors from $1+\mathcal{M}_{v}$. So

$$
\forall x^{\prime} \in U \exists z_{P_{k l}} \in \mathcal{M}_{v} \text { such that } P_{k l}\left(\bar{a}, x^{\prime}\right)=P_{k l}(\bar{a}, x) \cdot\left(1+z_{P_{k l}}\right) \text { etc. }
$$

However, a 1-unit factor does not affect the angular component or the value of an element. Hence, for all $k, l$

$$
\mathcal{K} \vDash \psi_{k l}(\bar{y}, \bar{\gamma}, \bar{a}, x) \leftrightarrow \psi_{k l}\left(\bar{y}, \bar{\gamma}, \bar{a}, x^{\prime}\right),
$$

and so $\varphi\left(\bar{a}, x^{\prime}\right)$ holds in $K$ as well. But then $\varphi(\bar{a}, x)$ does not define a finite set ( $U$ is infinite), a contradiction resulting from our assumption that $K$ is not slim. Hence $K$ is slim, after all.

Corollary 5.6 A (pure) henselian field of characteristic 0 has no proper definable subfields.

Proof: Theorem 5.5 and Proposition 4.1.

Example 5.7 If $K$ is a field of characteristic 0, then the formal power series field $K((X))$ is very slim in the ring language.

Question 7 Are all perfect henselian valued fields of positive characteristic very slim?

Question 8 Is $\mathbb{F}_{p}((X))^{\text {perf }}$ very slim?

\section{Examples}

\section{Slim, but not necessarily very slim fields}

Of course, all algebraic fields (that is, no transcendental elements at all) are slim, but in general not very slim.

Lemma 6.1 A PAC field is slim if it is a separable extension of all its relatively algebraically closed subfields.

Proof: Let $k \subseteq K$ be relatively algebraically closed. From [CMD] we know that $\operatorname{acl}_{K}(k)$ is the relative algebraic closure of the closure of $k$ unter the $\lambda$-functions of $K$. But $K$ is seperable over $k$ by assumption, hence $k$ is closed under the $\lambda$-functions.

Corollary 6.2 $\mathbb{F}_{p}(X)^{\mathrm{sep}}$ and $\mathbb{F}_{p}(X, Y)^{\mathrm{sep}}$ are slim.

Proof: The only relatively algebraically closed subfields of $\mathbb{F}_{p}(X)^{\text {sep }}$ are the field itself and $\widetilde{\mathbb{F}_{p}}$. The relatively algebraically closed subfields of $K:=\mathbb{F}_{p}(X, Y)^{\text {sep }}$ are $K, \widetilde{\mathbb{F}_{p}}$ and subfields $k$ of transcendence degree 1 . The latter are relative algebraic closures of functions fields $\mathbb{F}_{p}(R(X, Y))$ for some rational function $R$ in $X$ and $Y$, without $p$-th root in $K$. Now, if neither $X$ nor $Y$ constitutes a separating transcendence basis, then both have to appear only in $p$-th power in $R$. But then the $p$-th root of $R(X, Y)$ would be in $K$.

Alternative PROOF FOR $K:=\mathbb{F}_{p}(X)^{\mathrm{sep}}$ : Let $k \subseteq K$ be relatively algebraically closed. If $k$ has transcendence degree 1 , then $k=K$. Otherwise $k=\widetilde{\mathbb{F}_{p}}$. Every transcendental element $z \in K$ has infinite orbit in the rational function field $k\left(z^{-p^{n}}\right) \subseteq K$, where $z^{-p^{n}}$ is no $p$-th power, and every automorphism of $k\left(z^{-p^{n}}\right)$ has an extension to its separable clousre $K$. 
Example 6.3 If $K$ is infinite and algebraic over the prime field, then $K(X)$ is slim.

Proof: Let $k \subseteq K(X)$. If $k$ has transcendence degree 0 , then $\tilde{k} \cap K(X)=K$, and $K$ has thickness 0 in $K(X)$ as every element of $K(X) \backslash K$ has infinite orbit under $\operatorname{Aut}_{K}(K(X))$. If $k$ has transcendence degree 1 , then $\tilde{k} \cap K(X)=K(X)$.

\section{Big sizes}

Example 6.4 $\mathbb{Q}$ is slim, but not very slim, and has size $L$ with $\mathrm{BM}_{\mathbb{Q}}(0)=0$ and $\mathrm{BM}_{\mathbb{Q}}(1)=\infty$.

Proof: $\mathbb{Q}$ is slim, because there are no transcendental elements, but not very slim: The natural numbers and their exponentiation is definable in $\mathbb{Q}$ (Julia Robinson, Matyasevich). For a nonstandard integer $x$, the numbers $p^{x}$ for prime numbers $p$ are algebraically independent elements that are definable over $x$.

We have already seen that this implies that number fields, $\mathbb{Q}(X)$ and $R(X)$ for real closed fields $R$ have also size $\mathrm{L}$.

Lemma 6.5 If $K$ is large and of transcendence degree at least 2 , then $K(X)$ is not slim. If $K$ is in addition of infinite transcendence degree, then $K(X)$ has size $L$.

Proof: If $K$ is large, then $K$ is $\emptyset$-definable in $K(X)$, see [Koe]. Then use Proposition 4.1 and Lemma $4.12(\mathrm{~b})$.

In particular, $\mathbb{C}(X)$ is not slim (and has size $\mathrm{L}$ with $\mathrm{BM}_{\mathbb{C}(X)}(2)=\infty$ ).

Example 6.6 The following fields are not slim:

(a) Function fields over $\mathbb{F}_{p}$ in one variable (Theorem 1 in [Koe]).

(b) Function fields over $\mathbb{Q}$ in one variable and of genus greater than 1 (according to Falting's Theorem).

(c) Most function fields in one variable over an arbitrary field, namely if the conditions specified in Theorem 1 in [Koe] are satisfied.

\section{References}

[Ad] Hans Adler Explanation of independence, Dissertation, Albert-Ludwigs-Universität Freiburg 2005. http://arxiv.org/abs/math.LO/0511616

[Ax] James Ax "The elementary theory of finite fields", Ann. of Math. 88 (1968), pp. 239-271.

[CMD] Zoe Chatzidakis, Angus Macintyre, Lou Van den Dries "Definable sets over finite fields", J. Reine Angew. Math. 427 (1992), pp. 107-135.

[EP] Antonio Jose Engler, Alexander Prestel Valued fields, Springer 2005.

[HH] V. Harnik, L. Harrington "Fundamentals of forking", Annals of Pure and Applied Logic vol. 26 (1984), pp. 245-286.

[Ju] Markus Junker "A note on equational theories", J. Symbolic Logic 65 no. 4 (2000), pp. 1705-1712. 
[JuK] Markus Junker, Ingo Kraus "Theories with equational forking", J. Symbolic Logic 67 no. 1 (2002,) pp. 326-340.

[JWh] Moshe Jarden, William H. Wheeler "Model-complete theories of $e$-free Ax fields", J. Symbolic Logic 48 no. 4 (1983), pp. 1125-1129.

[Koe] Jochen Koenigsmann "Defining transcendentals in function fields", J. Symbolic Logic 67 no. 3 (2002), pp. 947-956.

[KP] B. Kim, A. Pillay "Simple Theories", Annals of Pure and Applied Logic, vol. 88 no. 2-3 (1997), pp. 149-164.

[Ons] Alf Onshuus "Properties and consequences of thorn-independence", J. Symbolic Logic 71 no. 1 (2006), pp. 1-21.

[Pas] Johan Pas "Uniform $p$-adic cell decomposition and local zeta functions", J. reine angew. Math. 399 (1989), pp. 137-172.

[Pop] Florian Pop "Embedding Problems Over Large Fields", The Annals of Mathematics, 2nd Series 144 no. 1 (1996), pp.1-34.

[PZ] Alexander Prestel, Martin Ziegler "Model theoretic methods in the theory of topological fields", J. reine angew. Math. 299/300 (1978), pp. 318-431.

[Rob1] Julia Robinson "The undecidability of algebraic rings and fields", Proc. Amer. Math. Soc. 10 (1959), pp. 950-957.

[Rob2] Julia Robinson "The decision problem for fields", in Theory of Models (Proc. 1963 Internat. Sympos. Berkeley) pp. 299-311, North-Holland, Amsterdam.

[Wh] William H. Wheeler "Model complete theories of pseudo-algebraically closed fields", Ann. Math. Logic 17 no. 3 (1979), pp. 205-226. 